\title{
Fabrication of a nano-sized ZSM-5 zeolite with intercrystalline mesopores for conversion of methanol to gasoline
}

\author{
Tingjun Fu, Jiangwei Chang, Juan Shao, Zhong Li* \\ Key Laboratory of Coal Science and Technology, Ministry of Education and Shanxi Province, \\ Taiyuan University of Technology, Taiyuan 030024, Shanxi, China
}

Article history:

Received 30 May 2016

Revised 5 September 2016

Accepted 7 September 2016

Available online

\begin{abstract}
Carbon deposition during methanol to hydrocarbons leads to the quick deactivation of ZSM-5 catalyst and it is one of the major problems for this technology. Decreasing the crystal size or introducing mesopores into ZSM-5 zeolites can improve its diffusion property and decrease the coke formation. In this paper, nano-sized ZSM-5 zeolite with intercrystalline mesopores combining the mesoporous and nano-sized structure was fabricated. For comparison, the mesoporous ZSM-5 and nano-sized ZSM-5 were also prepared. These catalyst samples were characterized by XRD, BET, $\mathrm{NH}_{3}$-TPD, TEM, Py-IR and TG techniques and used on the conversion of methanol to gasoline in a fixed-bed reactor at $T=405^{\circ} \mathrm{C}$, WHSV $=4.74 \mathrm{~h}^{-1}$ and $P=1.0$ MPa. It was found that the external surface area of the nano-sized ZSM-5 zeolite with intercrystalline mesopores reached $104 \mathrm{~m}^{2} / \mathrm{g}$, larger than that of mesoporous ZSM-5 $\left(66 \mathrm{~m}^{2} / \mathrm{g}\right)$ and nano-sized ZSM-5 $\left(76 \mathrm{~m}^{2} / \mathrm{g}\right)$. Catalytic lifetime of the nano-sized ZSM-5 zeolite with intercrystalline mesopores was $93 \mathrm{~h}$, which was only longer than that of mesoporous ZSM-5 (86 h), but shorter than that of nano-sized ZSM-5 (104 h). Strong acidity promoted the coke formation and thus decreased the catalytic lifetime of the nano-sized ZSM-5 zeolite with intercrystalline mesopores though it presented large external surface that could improve the diffusion property. The special zeolite catalyst was further dealuminated to decrease the strong acidity. After this, its coke formation rate was slowed and catalytic lifetime was prolonged to $106 \mathrm{~h}$ because of the large external surface area and decreased weak acidity. This special structural zeolite is a potential catalyst for methanol to gasoline reaction.
\end{abstract}

Keywords: ZSM-5; Crystal size; Mesopore; Acidity; Methanol to gasoline

*Corresponding author. Tel/Fax: +86 3516018526; E-mail: lizhong@tyut.edu.cn.

This work was supported by the Science and Technology Foundation Platform Construction Project of Shanxi Province (No. 2015091009), the National Science Foundation for Young 
Scientists of China (No. 21606160), the Qualified Personnel Foundation of Taiyuan University of Technology (No. tyut-rc201454a) and School Fund of Taiyuan University of Technology (No.1205-04020202; No.1205-04020102).

\section{Introduction}

As a result of the increasing global demand on the transport fuel and the adverse effects of fluctuating energy price, searching for alternatives to crude oil has become one of the most important issues in current society $[1,2]$. This situation is more crucial for the country whose petroleum consumption strongly depends on the importation. Among possible alternatives, methanol to gasoline (MTG) technology is currently gaining more and more attentions because feedstock methanol is readily produced from synthesis gas through gasification of coal, biomass, wastes or steaming reforming of natural gas, and the obtained methanol can be catalytically converted to high octane gasoline [3,4]. For this technology, ZSM-5 zeolite has been proved to be the major catalyst that can complete above conversion, due to its well-defined micropores, large surface area, strong acidity and high hydrothermal stability [5]. However, it is the well-defined micropores that seriously restrict the diffusion of bulk molecules, which slows down the diffusion of the coke precursors from micropores to the external surface. These coke precursors could polymerize within the micropores to form coke and then lead to the rapid catalyst deactivation due to the coverage on strong acid sites and blocking of micropores [6-9]. Coke deposition of ZSM-5 has been deemed as one key problem to be solved for MTG technology.

Generally, two most promising strategies are being investigated to solve this problem. One is to decrease the crystal size of ZSM-5 zeolite, which has been recognized as an important way to improve the catalytic performance in methanol to hydrocarbon (MTH) reactions. Rownaghi et al. [10] found that nano ZSM-5 with crystal size of $120 \mathrm{~nm}$ realized a complete conversion of methanol at the first $10 \mathrm{~h}$ of the reaction, while the conversion of methanol on micrometer ZSM-5 was only $65 \%$ and decreased quickly to $60 \%$ after only $5 \mathrm{~h}$ of reaction. Firoozi et al. [11] investigated the effects of crystal size of ZSM-5 on the methanol to propene performance and found that during $200 \mathrm{~h}$ reaction of testing, methanol conversion on nano-sized ZSM-5 was always higher than that on micro-sized ZSM-5. The high catalytic activity and long lifetime of the small sized ZSM-5 were attributed to its decreased diffusion length and more available acid sites. However, the synthesis of nano-sized ZSM-5 is far from industrial production due to long crystallization time, low yield and large consumption of expensive organic directing agents (ODA), which could increase the manufacturing costs and cause air pollution during the process of removing templates. In addition, the separation using high-speed centrifugation and the low crystallinity of nano-sized ZSM-5 also restrict its application on a large scale. Currently, the search of simple and economical preparation methods for nano-sized ZSM-5 is still a key issue for its practical application. 
Another approach to solve the coke problem is to fabricate mesopores on ZSM-5. Alkaline desilication is a simple and efficient means. These mesopores are mainly produced inside the ZSM-5 crystal and their positive influence on the catalytic performance has been evidenced in MTH reaction. Fathi et al. [12] studied the effects of alkaline types $\left(\mathrm{CaCO}_{3}, \mathrm{Na}_{2} \mathrm{CO}_{3}\right.$ and $\left.\mathrm{NaOH}\right)$ on mesopores formation and found that desilicated ZSM-5 by $\mathrm{Na}_{2} \mathrm{CO}_{3}$ solution showed greater improvement in catalytic lifetime and the yield of gasoline hydrocarbons. The effects of the concentration of $\mathrm{NaOH}$ solution on the pore structure of ZSM-5 and its MTG performance was also investigated by Bjørgen et al. [13]. It was found that the catalytic lifetime of the sample treated by $0.05 \mathrm{M}$ alkaline solution was prolonged from 35 to $75 \mathrm{~h}$ at the conditions of $370{ }^{\circ} \mathrm{C}$ and WHSV $=8 \mathrm{~h}^{-1}$. Enhanced catalytic performance and coke-resistance have also been observed for mesoporous ZSM-5 obtained by $\mathrm{NaOH}$ post-treatment in methane aromatization reaction and the beneficial effects were attributed to the formation of mesopores and improved mass transfer efficiency [14]. However, it is known that the extraction of Si during desilication process is ruled by the framework $\mathrm{Al}$ [15]. The presence of framework $\mathrm{Al}$ in ZSM-5 zeolite prevents the Si from being extracted. The nonuniform distribution of framework $\mathrm{Al}$ induces excessive $\mathrm{Si}$ extraction in the interior of crystal during desilication process and results in large voids or even broken crystal pieces, which is not beneficial to the enhancement of diffusion property. Also, the extraction of $\mathrm{Si}$ during desilication process causes a waste of synthetic raw material and results in a low yield of treated products.

Salt-aided seed-induced route has been recently proposed to synthesize nano-sized ZSM-5 with intercrystalline mesopores. With the aid of seeds, the crystallization process was fast and the yield of products could reach $85 \%$. Interestingly, the gel-rich system with $\mathrm{Na}^{+}, \mathrm{K}^{+}, \mathrm{Br}^{-}$and $\mathrm{SO}_{4}{ }^{2-}$ ions causes the salting out of aluminosilicates, which results in abundant intercrystalline mesopores by the assembly of adjacent nanocrystallines [16,17]. The in situ formation of mesopores replaces the desilication process and avoids the waste of synthetic raw material. The difficulty of separation of nano-sized ZSM-5 after crystallization is also solved due to the aggregated structure formed by stacking of the nano-sized crystallites. The most important thing is that nano-sized ZSM-5 with intercrystalline mesoporous structure possesses the advantages of both mesoporous ZSM-5 and nano-sized ZSM-5 (as shown in Fig. 1). Moreover, the intercrystalline mesoporous ZSM-5 has rich strong acid sites, although it is composed of a large number of nanocrystallines, which usually presents weak acidity due to the framework defects [18]. 


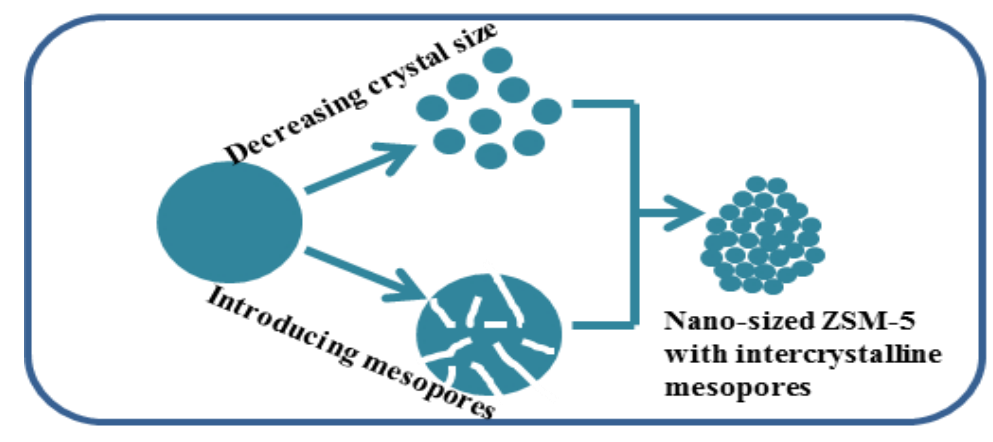

Fig. 1. Schematic diagram of nano-sized ZSM-5 with intercrystalline mesopores.

In view of the significant influence of mesopore and crystal size of ZSM-5 on the catalytic performance, nano-sized ZSM-5 with intercrystalline mesopores are expected to display an excellent activity in MTG reactions. However, there has not been any research about the application of nano-sized ZSM-5 with intercrystalline mesopores on MTG reaction, and the influence mechanism of the composite structure formed with nano-size crystals and mesopores on the catalytic activity remains unclear. In this work, nano-sized ZSM-5 with intercrystalline mesopores was prepared by salt-aided seed-induced route. In comparison, nano-sized ZSM-5 and mesoporous ZSM-5 were also prepared. The catalytic performance of the above catalysts on MTG reaction was studied. Concerning the strong acidity of nano-sized ZSM-5 with intercrystalline mesopores would accelerate the formation of coke species and shorten the catalytic lifetime, it was dealuminated to reduce the acidity to increase the longevity. Based on this research, we expect to find a new ZSM-5 catalyst with good MTG performance.

\section{Experimental}

\subsection{Catalyst preparation}

ZSM-5 catalyst with average crystal size of $500 \mathrm{~nm}$ was synthesized from a clear solution according to the procedure reported by Mochizuki et al. [19]. The molar composition of the starting gel was $1 \mathrm{SiO}_{2}: 0.017 \mathrm{Al}_{2} \mathrm{O}_{3}: 0.25$ TPAOH:0.05 Na2 $\mathrm{O}: 60 \mathrm{H}_{2} \mathrm{O}$. Firstly, required tetraethyl orthosilicate (TEOS, $98 \mathrm{wt} \%$ ), tetrapropylammonium hydroxide (TPAOH, $25 \mathrm{wt} \%$ ) and water were mixed under stirring at $80{ }^{\circ} \mathrm{C}$ for $24 \mathrm{~h}$. Then, the solution containing water, $\mathrm{Al}\left(\mathrm{NO}_{3}\right)_{3} \cdot 9 \mathrm{H}_{2} \mathrm{O}$ and $\mathrm{NaOH}$ was added to the above clear mixture. The prepared gel was transferred to a $300 \mathrm{~mL}$ Teflon lined steel autoclave and crystallized at $170{ }^{\circ} \mathrm{C}$ for $24 \mathrm{~h}$. The products were then recovered by centrifugation, washed with deionized water and dried at $120{ }^{\circ} \mathrm{C}$ overnight. Thereafter, Na-ZSM-5 sample was obtained by calcination at $550{ }^{\circ} \mathrm{C}$ for $6 \mathrm{~h}$ to remove $\mathrm{TPA}^{+}$species. H-form zeolite was prepared by ion-exchanged with $0.8 \mathrm{M} \mathrm{NH}_{4} \mathrm{NO}_{3}\left(20 \mathrm{~mL} / \mathrm{g}\right.$-sample) for $3 \times 8 \mathrm{~h}$ at $80{ }^{\circ} \mathrm{C}$ followed by calcination at $550{ }^{\circ} \mathrm{C}$ for $6 \mathrm{~h}$. The obtained sample is designated as Z5-500. Nano-sized ZSM-5 with average crystal size of $70 \mathrm{~nm}$ was also synthesized by the similar process 
and the molar composition of the starting gel was $1 \mathrm{SiO}_{2}: 0.017 \mathrm{Al}_{2} \mathrm{O}_{3}: 0.25$ TPAOH:0.05 Na2 $\mathrm{O}: 8.3$ $\mathrm{H}_{2} \mathrm{O}$. The obtained sample is designated as Z5-70.

ZSM-5 with intracrystalline mesopores was prepared by mixed alkaline treatment (TPAOH and $\mathrm{NaOH}$ ) of Z5-500. During the process, the use of $\mathrm{TPA}^{+}$could protect the Si extraction and avoid the formation of large pores or hollow structure caused by the excessive desilication. Typically, the desilication process of Z5-500 was carried out in a $0.2 \mathrm{M}$ mixed alkaline solution (the mole ratio of $\left.\mathrm{TPA}^{+} / \mathrm{OH}^{-}=0.25\right)$ solution $\left(15 \mathrm{~mL} / \mathrm{g}\right.$-zeolite) under vigorous stirring at $50{ }^{\circ} \mathrm{C}$ for $2 \mathrm{~h}$ then cooled in ice water immediately, followed by filtration and washing with de-ionized water until neutral. The solid products were dried overnight at $120{ }^{\circ} \mathrm{C}$ and then calcinated at 550 ${ }^{\circ} \mathrm{C}$ for $6 \mathrm{~h}$. H-form zeolite was obtained by ion-exchanged with $0.8 \mathrm{M} \mathrm{NH}_{4} \mathrm{NO}_{3}(20 \mathrm{~mL} / \mathrm{g}$-zeolite) for $3 \times 8 \mathrm{~h}$ at $80{ }^{\circ} \mathrm{C}$ followed by calcination at $550{ }^{\circ} \mathrm{C}$ for $6 \mathrm{~h}$. The obtained sample is designated as Z5-500-Si.

Nano-sized ZSM-5 with intercrystalline mesopores was synthesized by the "salt-aided seed-induced route" [16,17]. For the typical synthesis process, a starting aluminosilicate gel with the molar ratio of $1 \mathrm{SiO}_{2}: 0.017 \mathrm{Al}_{2} \mathrm{O}_{3}: 0.15 \mathrm{Na}_{2} \mathrm{O}: 0.1 \mathrm{TPABr}: 0.3 \mathrm{KF}: 30 \mathrm{H}_{2} \mathrm{O}$ was prepared. The procedure was simply described as follows: the solution of $\mathrm{NaOH}, \mathrm{Al}_{2}\left(\mathrm{SO}_{4}\right)_{3} \cdot 9 \mathrm{H}_{2} \mathrm{O}, \mathrm{KF}$ and $\mathrm{TPABr}$ were mixed well under vigorous stirring, then colloidal silica was added dropwise by plunger pump. Thereafter, the colloidal solution of silicate-1 seed was added to the above solution and the addition quantity equaled to $7.0 \mathrm{wt} \%$ of total $\mathrm{SiO}_{2}$ weight in the starting gel. The colloidal solution of silicate-1 seed was synthesized by a clear solution method [20]. The prepared gel was transferred to a $300 \mathrm{~mL}$ Teflon lined steel autoclave and crystallized at $120{ }^{\circ} \mathrm{C}$ for $24 \mathrm{~h}$. The products were then centrifugated, washed with deionized water and dried at $120{ }^{\circ} \mathrm{C}$ overnight. Thereafter, Na-ZSM-5 sample was obtained by calcination at $550{ }^{\circ} \mathrm{C}$ for $6 \mathrm{~h}$ to remove $\mathrm{TPA}^{+}$ species. Then $\mathrm{H}$-form zeolite was prepared by ion-exchanged with $0.8 \mathrm{M} \mathrm{NH}_{4} \mathrm{NO}_{3}$ (20 $\mathrm{mL} / \mathrm{g}$-sample) for $3 \times 8 \mathrm{~h}$ at $80{ }^{\circ} \mathrm{C}$ followed by calcination at $550{ }^{\circ} \mathrm{C}$ for $6 \mathrm{~h}$. The obtained sample is designated as Z5-inter.

In order to improve the catalytic performance, Z5-inter was dealuminated with $0.2 \mathrm{M} \mathrm{HCl}$ (15 mL/g-zeolite) under vigorous stirring at $50{ }^{\circ} \mathrm{C}$ for $2 \mathrm{~h}$ and then cooled in ice water immediately, followed by filtration and washing with de-ionized water until neutral. The solid products were dried overnight at $120{ }^{\circ} \mathrm{C}$ and then calcinated at $550{ }^{\circ} \mathrm{C}$ for $6 \mathrm{~h}$. The obtained sample is designated as Z5-inter-Al.

\subsection{Catalyst characterization}

$\mathrm{X}$-ray diffraction (XRD) equipped with $\mathrm{Cu} K \alpha$ radiation was used to identify the phase and calculate the crystallinity of the samples. Operations were performed at $40 \mathrm{kV}$ and $40 \mathrm{~mA}$. The patterns were scanned at a rate of $8 \% \mathrm{~min}$ in the range $2 \theta=5^{\circ}-50^{\circ}$. 
The information about the morphology and mesopores of prepared samples were studied by transmission electron microscopy (TEM, JEM-2100F). All samples subjected to TEM were ultrasonically dispersed in ethanol for $30 \mathrm{~min}$ and then dropped on copper grids.

The physical textural properties of catalysts were performed by $\mathrm{N}_{2}$ adsorption-desorption isotherms obtained at $77 \mathrm{~K}$ using Beishide 3H-2000PS2 apparatus. Prior to the measurement, the samples were degassed under high vacuum at $250{ }^{\circ} \mathrm{C}$ for $4 \mathrm{~h}$. The surface area was obtained using Brunauer-Emmett-Teller (BET) model for adsorption data in a relative pressure range of $P / P 0=0.05-0.30$. The total pore volumes were calculated from the amount of $\mathrm{N}_{2}$ vapor adsorbed at a relative pressure of 0.99 .

Temperature-programmed desorption of $\mathrm{NH}_{3} \quad\left(\mathrm{NH}_{3}-\mathrm{TPD}\right)$ was performed out on MicromeriticsAutoChem 2920 chemisorption analyzer to determine the quantity and strength of acid sites of the catalysts. $0.1 \mathrm{~g}$ of sample was firstly preheated in flowing helium at $550{ }^{\circ} \mathrm{C}$ for 1.5 $\mathrm{h}$ and then cooled down to $120{ }^{\circ} \mathrm{C}$, and each sample was then kept in $15 \mathrm{vol} \% \mathrm{NH}_{3} / \mathrm{He}$ flowing $(40$ $\mathrm{mL} / \mathrm{min}$ ) for $30 \mathrm{~min}$ until the saturated ammonia adsorption was attained. Then the flowing gas was switched to pure $\mathrm{He}$ for $1 \mathrm{~h}$ to remove the physically adsorbed $\mathrm{NH}_{3}$ molecules. The temperature programmed desorption of the chemically adsorbed $\mathrm{NH}_{3}$ was monitored by a thermal conductivity detector by a constant heating rate of $10{ }^{\circ} \mathrm{C} / \mathrm{min}$ from 120 to $650{ }^{\circ} \mathrm{C}$.

FT-IR spectra of adsorbed pyridine were recorded on a Bruker Tensor 27 spectrometer. The sample powder was pressed into a self-supporting wafer (diameter: $13 \mathrm{~mm}$; weight: $20 \mathrm{mg}$ ) and activated at $200{ }^{\circ} \mathrm{C}$ for 30 min under vacuum $\left(10^{-2} \mathrm{~Pa}\right)$. Probe molecule adsorption was taken place in situ at room temperature, followed by evacuation at $200{ }^{\circ} \mathrm{C}$ for $15 \mathrm{~min}$. Afterwards an IR spectrum was recorded at room temperature and a difference spectrum was obtained by subtracting the spectrum of the activated sample from the spectrum after probe adsorption.

The content of coke species in the deactivated ZSM-5 after MTG reaction was determined by TG (Setaram SETSYS Evolution TGA) characterization. About $10 \mathrm{mg}$ of the sample was used and heated to $700{ }^{\circ} \mathrm{C}$ by a heating rate of $10{ }^{\circ} \mathrm{C} / \mathrm{min}$. The coke content was calculated in percent of the mass of coke free catalyst.

\subsection{Catalyst evaluation}

Methanol to gasoline reaction was carried out in a stainless-steel fixed bed reactor (12 mm ID, $110 \mathrm{~cm}$ length). Before reaction, a mixture of $1.0 \mathrm{~g}$ pressed HZSM- 5 catalysts (80-100 mesh) and $2.0 \mathrm{~g}$ quartz sands (20-40 mesh) was loaded in the center of the reactor. A K-type thermocouple was placed into the center of the catalysts bed and the average temperature of the catalyst bed was kept at $405^{\circ} \mathrm{C}$. Before the reaction, the catalysts mixed with quartz sands were activated in situ at $450{ }^{\circ} \mathrm{C}$ for $1.5 \mathrm{~h}$ in $\mathrm{N}_{2}$ flow. After catalyst activation, the temperature was decreased to $400{ }^{\circ} \mathrm{C}$ and maintained under $\mathrm{N}_{2}$ atmosphere $(35 \mathrm{~mL} / \mathrm{min}$ ). The pressure of system was maintained at $1.0 \mathrm{MPa}$ by adjusting the back pressure regulator. Then a plunger pump was used to feed methanol at a flow of $0.1 \mathrm{~mL} / \mathrm{min}$ following the $\mathrm{N}_{2}$ flow to start the process. After the reaction, a gas/liquids 
separator at ca. $1.0{ }^{\circ} \mathrm{C}$ was subsequently located to collect liquid hydrocarbons and water. After separation from water, liquid hydrocarbons were weighed to calculate its density and then off-line analyzed on a SHIMADZU (GC-2014C) chromatograph that was equipped with a $35 \mathrm{~m}$ capillary column and FID using $\mathrm{N}_{2}$ as carrier gas.

\section{Results and discussion}

\subsection{Characterization of the catalysts}

The powder XRD patterns of the zeolite catalysts prepared by different methods were shown in Fig. 2. It can be seen that all samples exhibited well-resolved diffraction peaks, which was consistent with typical patterns of MFI crystalline structure [21]. Visual inspection obviously showed that Z5-500 with crystal size of $500 \mathrm{~nm}$ had the strongest diffraction peak intensity. In order to expediently discuss the differences of crystallinity for these ZSM-5 samples, the crystallinity of Z5-500 was defined as $100 \%$. In comparison, the relative crystallinity of nano-sized Z5-70 with crystal size of $70 \mathrm{~nm}$ was $80 \%$, probably as a result of more framework defects exposed on the external surface with the decrease of crystal size [22,23]. For Z5-500-Si obtained by alkaline treatment of Z5-500, its relative crystallinity was decreased to $85 \%$, indicating the partial damage of framework structure after desilication [12]. It is interesting that Z5-inter with intracrystalline mesopores presented broad and weak diffraction peaks and its relative crystallinity was only $45 \%$. The low relative crystallinity of Z5-inter was closely related to the synthesis condition. During its synthesis process, a large amount of ions, such as $\mathrm{Na}^{+}, \mathrm{K}^{+}, \mathrm{Br}^{-}$ and $\mathrm{SO}_{4}{ }^{2-}$ were introduced into the gel to form a gel-rich system. In this system, the salting out and crystallization of aluminosilicates happened simultaneously during its crystallization process and the intercrystalline mesopores were formed at the cost of the topology structure. After acid treatment, Z5-inter-Al showed a relative crystallinity of $60 \%$, higher than that of Z5-inter. The reasons might be that some defect structures were eliminated during the process. Although the reduction of crystal size and the creation of mesopores were two possible ways to improve diffusion property of ZSM-5, the crystallinity was lowered due to the damage of microporous structure as discussed above. Especially for Z5-inter, the creation of intercrystalline mesopores was mainly at the cost of microporous structure.

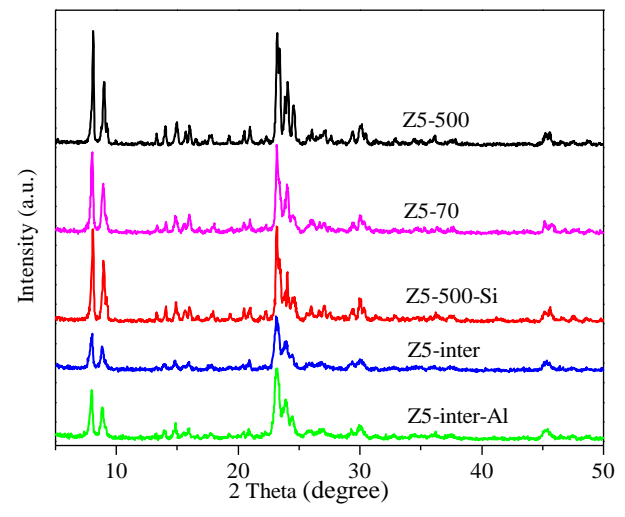

Fig. 2. XRD patterns of the ZSM-5 zeolites. 
Table 1. Relative crystallinity of the ZSM-5 zeolites.

\begin{tabular}{|c|c|c|c|c|c|}
\hline Catalyst & Z5-500 & $\mathrm{Z5}-70$ & Z5-500-Si & Z5-inter & Z5-inter-Al \\
\hline Relative crystallinity $^{*}(\%)$ & 100 & 80 & 85 & 45 & 60 \\
\hline
\end{tabular}

The morphology and mesoporous structure of the above catalysts were investigated by TEM characterization. Fig. 3 showed the obvious structure differences among these catalysts. Z5-500 and Z5-70 (Fig. 3a, b) exhibited uniform monodispersed crystals with an average size of 500 and $70 \mathrm{~nm}$, respectively. In this work, these two different-sized ZSM-5 catalysts were prepared by changing the $\mathrm{H}_{2} \mathrm{O} / \mathrm{Si}$ ratio in the initial synthesis gel. The $\mathrm{H}_{2} \mathrm{O} / \mathrm{Si}$ ratio in the synthesis solution for Z5-70 was 8.3 and that for Z5-500 was 60. Less water in the solution can cause a higher alkalinity condition, which favors the nucleation and formation of small-sized ZSM-5 [20]. Also, the surface of Z5-500 and Z5-70 was smooth and no evident large bright spots relative to large mesopores were observed. On the contrary, the images of Z5-500-Si sample prepared by alkaline-treatment showed bright spots. These bright fields were the evidence of formation of intracrystalline mesopores due to the extraction of framework Si during the alkaline treatment. Alkaline treatment usually leads to uncontrollable desilication to form large voids or even hollow structure [15]. In this work, ZSM-5 was desilicated using mixed alkaline solution of $\mathrm{NaOH}$ and TPAOH. This treating method not only induced the formation of mesopores, but also well-preserved the integrated structure and avoided the formation of hollow structure. According to the images in Fig.3(d), Z5-inter possessed globular structure with the crystal size of ca. $500 \mathrm{~nm}$ and the particles were composed by oriented intergrowth of lots of small nanocrystals. The in situ assembly of these nanocrystals gave rise to abundant intercrystalline mesopores (red circles in Fig. 3d) [24]. After acid treatment, the sample Z5-inter-Al showed no obvious changes on morphology and structure. 

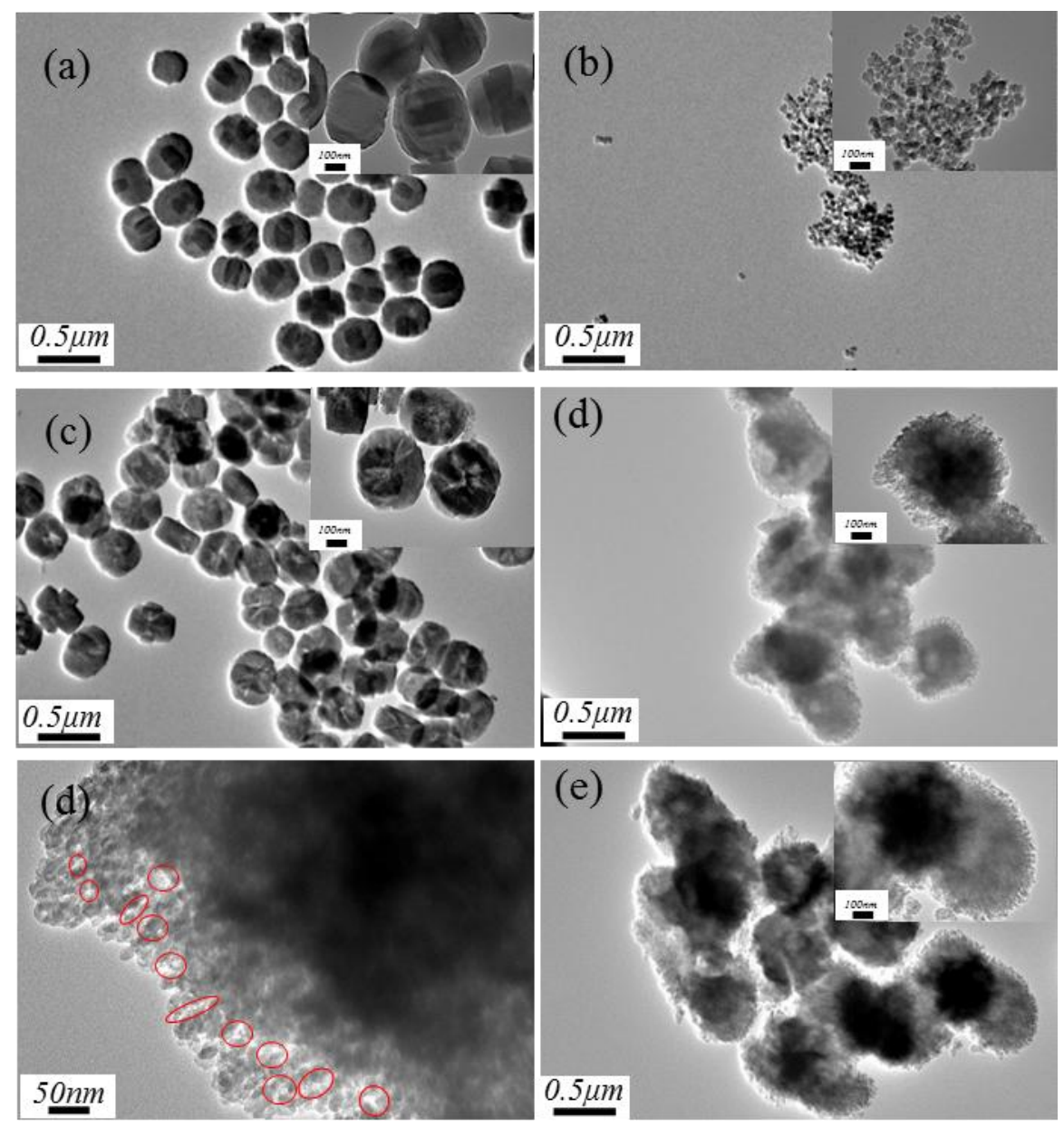

Fig. 3. TEM images of (a) Z5-500, (b) Z5-70, (c) Z5-500-Si, (d) Z5-inter, and (e) Z5-inter-Al.

The structural and textural properties of the catalysts were also characterized by $\mathrm{N}_{2}$ sorption. As shown in Fig. 4, the samples Z5-500 and Z5-70 displayed type I isotherms and only presented uptake at low relative pressure $\left(P / P_{0}<0.2\right)$, implying the microporous structure for these two samples. But for Z5-500-Si, the isotherm was corresponded to type IV and the hysteresis loop showed a type-H4 with a steep down on desorption around $P / P_{0}=0.45$, which was ascribed to the cavitation effect of ink-bottle type of intracrystalline mesopores formed by alkali desilication. Z5-inter sample showed a type IV isotherm exhibiting a type-H3 hysteresis loop, which arose from the nitrogen adsorption on the intercrystalline voids by assembly of adjacent nano-crystallites [16,24-26]. After dealumination, Z5-inter-Al possessed a similar hysteresis loop with Z5-inter. The textural properties of the samples were summarized and shown in Table 2. It can be seen that the mesopore volume and external surface area of Z5-500 were $0.15 \mathrm{~cm}^{3} / \mathrm{g}$ and 40 $\mathrm{m}^{2} / \mathrm{g}$, respectively. For Z5-70 zeolite, the external surface area reached $76 \mathrm{~m}^{2} / \mathrm{g}$, higher than that of Z5-500. But the mesopore volume was only $0.13 \mathrm{~cm}^{3} / \mathrm{g}$, similar to that of Z5-500, indicating the well maintained micropores of Z5-70. Considering the absence of meso- or macropores, the large external surface area for Z5-70 was ascribed to the decrease of crystal size. For Z5-500-Si sample, desilication facilitated the formation of intracrystalline mesopore and thus the mesopore volume 
was obviously increased to $0.35 \mathrm{~cm}^{3} / \mathrm{g}$. Correspondingly, the external surface area was improved to $66 \mathrm{~m}^{2} / \mathrm{g}$. The surprising thing is that Z5-inter, a nano-sized ZSM-5 with intercrystalline mesopores, presented an external surface area of $104 \mathrm{~m}^{2} / \mathrm{g}$, larger than that of Z5-70. But the micropore volume was similar to Z5-70, which also proved that Z5-inter still maintained the microporous structure. After dealumination treatment, the external surface area of obtained Z5-inter-Al was slightly decreased to $100 \mathrm{~m}^{2} / \mathrm{g}$, indicating the well maintained pore structure (Fig. $3 \mathrm{e})$.

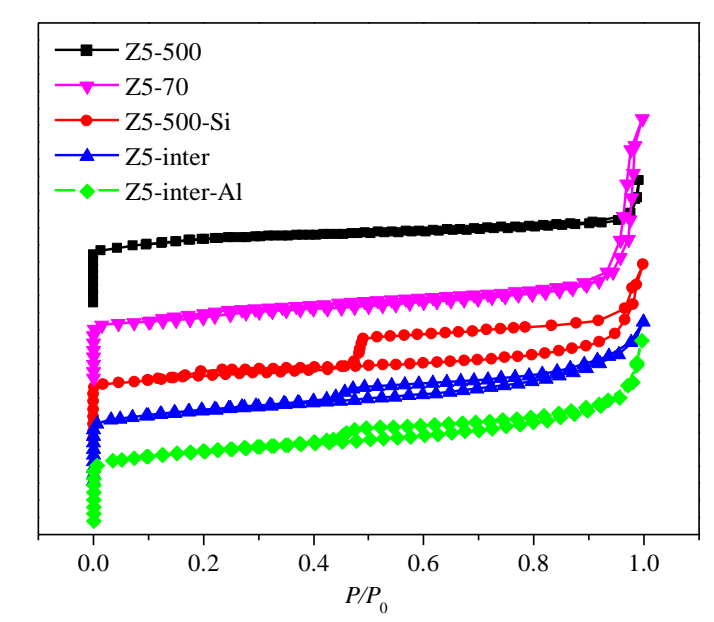

Fig. 4. $\mathrm{N}_{2}$ adsorption and desorption isotherms of different ZSM-5 zeolites.

Table 2. Textural properties of different ZSM-5 zeolites.

\begin{tabular}{llllll}
\hline Catalyst & $\begin{array}{c}S_{\text {BET }}^{\mathrm{a}} \\
\left(\mathrm{m}^{2} / \mathrm{g}\right)\end{array}$ & $\begin{array}{c}S_{\text {ext }}^{\mathrm{b}} \\
\left(\mathrm{m}^{2} / \mathrm{g}\right)\end{array}$ & $\begin{array}{c}S_{\text {micro }}^{\mathrm{b}} \\
\left(\mathrm{cm}^{2} / \mathrm{g}\right)\end{array}$ & $\begin{array}{c}V_{\text {micro }}^{\mathrm{b}} \\
\left(\mathrm{cm}^{3} / \mathrm{g}\right)\end{array}$ & $\begin{array}{l}V_{\text {meso }}^{\mathrm{c}} \\
\left(\mathrm{cm}^{3} / \mathrm{g}\right)\end{array}$ \\
\hline Z5-500 & 345 & 40 & 305 & 0.17 & 0.15 \\
Z5-70 & 380 & 76 & 304 & 0.14 & 0.13 \\
Z5-500-Si & 367 & 66 & 301 & 0.14 & 0.35 \\
Z5-inter & 391 & 104 & 287 & 0.13 & 0.29 \\
Z5-inter-Al & 374 & 100 & 274 & 0.14 & 0.28 \\
\hline
\end{tabular}

${ }^{a}$ Derived from the BET model. ${ }^{b}$ By $t$-plod method. ${ }^{c}$ Using the BJH method by desorption. $S_{\text {ext }}$ : external surface area. $V_{\text {micro }}$ : micropore volume. $V_{\text {meso }}$ : mesopore volume.

Moreover, as indicated by the pore size distribution curves in Fig. 5, it can be seen that these catalysts presented different pore size distributions. The Z5-500 has only small pores smaller than $3 \mathrm{~nm}$, which should be formed by desilication resulted from the strong alkalinity of synthesis solution. Z5-70 also presented some mesopores smaller than $4 \mathrm{~nm}$ formed during synthesis process. Except these mesopores, some mesopores with the relative larger diameter centered at ca. 
$40 \mathrm{~nm}$ can also be found, probably rooting in intercrystalline voids formed by the aggregation of nanocrystallites. For Z5-500-Si, abundant mesopores with concentrated distribution at $2 \mathrm{~nm}$ existed, indicating the formation of the uniform mesoporous structure. Unlike above mentioned three catalysts, the Z5-inter has abundant mesopores with 2-10 nm, which were formed by the in situ assembly of these nanocrystals. After acid treatment, the sample Z5-inter-Al showed no obvious change on pore size distribution.

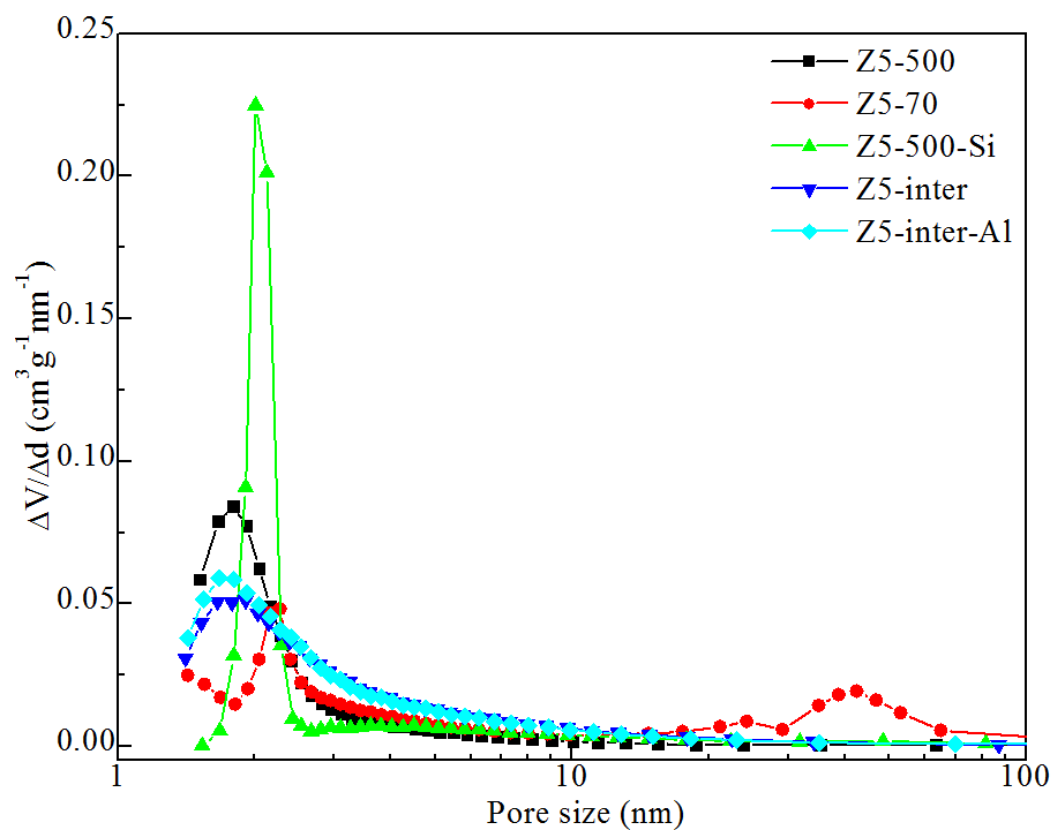

Fig. 5. BJH pore size distributions of the ZSM-5 samples deduced from the adsorption brand of isotherms.

The amount and strength of acid sites of the samples were investigated by $\mathrm{NH}_{3}$-TPD characterization. After the deconvolution and ammonia quantification, each $\mathrm{NH}_{3}$ - TPD curve was divided by Gauss curve fitting method into three separated peaks. As shown in Fig. 6, all the samples presented three $\mathrm{NH}_{3}$-TPD curves with different peak temperatures, which were assigned to the weak, medium and strong acid sites, respectively. The amounts of these three types of acid sites were calculated and summarized in Table 3. The amount of acid sites of Z5-500 was 0.65 $\mathrm{mmol} / \mathrm{g}$, and that of Z5-70 was only $0.58 \mathrm{mmol} / \mathrm{g}$. Also, the peak temperatures of both weak and strong acid sites of Z5-70 were shifted to lower temperature compared with Z5-500, which might benefit from its lower crystallinity with more framework defects [13,22,27], as shown by XRD and $\mathrm{N}_{2}$ adsorption/desorption results. So it could be found that the zeolite acidity was decreased when the crystal size was reduced based on above comparison of the amount of acid sites and the peak temperatures in $\mathrm{NH}_{3}-\mathrm{TPD}$ profiles. For $\mathrm{Z} 5-500-\mathrm{Si}$, all the peak temperature positions were shifted to higher temperature compared with Z5-500. The amount of weak and strong acid sites was also improved by $0.09 \mathrm{mmol} / \mathrm{g}$ and $0.04 \mathrm{mmol} / \mathrm{g}$, respectively. This indicated the improvement of acidity because of the increased Al content, which was attributed to the preferential desilication during alkali treatment. Z5-inter with intercrystalline mesopores presented 
strong acidity, the total acid amount and the number of strong acid sites was much more than other catalysts and reached up to $1.29 \mathrm{mmol} / \mathrm{g}$ and $0.58 \mathrm{mmol} / \mathrm{g}$, respectively. The peak temperatures of both weak and strong acid sites were also higher than other catalysts. To further investigate the effects of strong acid sites on catalytic lifetime, Z5-inter was dealuminated to reduce the amount of strong acid sites. The peak temperatures of both strong and weak acid sites were shifted to lower temperature and the amount of acid sites was decreased, especially the strong acid sites.

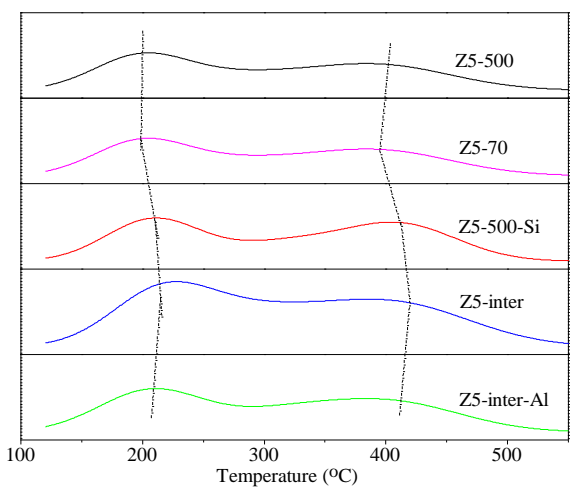

Fig. 6. $\mathrm{NH}_{3}$-TPD patterns of ZSM-5 zeolites.

Table 3. Acidity of different ZSM-5 zeolites.

\begin{tabular}{lcccc}
\hline \multirow{2}{*}{ Samples } & \multicolumn{4}{c}{ Distribution of acid sites (mmol/g) } \\
\cline { 2 - 5 } & Total & Weak $\left(150-250{ }^{\circ} \mathrm{C}\right)$ & Medium $(250-350$ & Strong $(350-450$ \\
& & & $\left.{ }^{\circ} \mathrm{C}\right)$ & 0.29 \\
\hline Z5-500 & 0.65 & 0.23 & 0.13 & 0.26 \\
Z5-70 & 0.58 & 0.17 & 0.15 & 0.33 \\
Z5-500-Si & 0.81 & 0.32 & 0.16 & 0.58 \\
Z5-inter & 1.29 & 0.40 & 0.31 & 0.29 \\
Z5-inter-Al & 0.71 & 0.28 & 0.14 & $\left.{ }^{\circ} \mathrm{C}\right)$ \\
\hline
\end{tabular}

Py-IR measurement were performed at desorption temperature of $200{ }^{\circ} \mathrm{C}$ to investigate the acid site distributions of the different samples. The results are shown in Fig. 7. In general, the two peaks appeared at around 1450 and $1540 \mathrm{~cm}^{-1}$ correspond to the characteristic vibration peaks of pyridine molecules adsorbed on Lewis and Brönsted acid sites, respectively. For a desorption temperature of $200{ }^{\circ} \mathrm{C}$, there were fewer Lewis acid sites $\left(1456 \mathrm{~cm}^{-1}\right)$ and more Brönsted acid sites $\left(1545 \mathrm{~cm}^{-1}\right)$ present on all the samples, showing that most of the acid sites on these samples were Brönsted acid type that is more crucial to MTH reaction than Lewis acid type. The distributions of Brönsted and Lewis acid sites on the different ZSM-5 zeolites are listed in Table 4. Specifically, the nano-sized Z5-70 possessed both more Brönsted and Lewis acid sites and little larger B/L ratio 
than Z5-500. After desilication of Z5-500, Z5-500-Si showed both the decrease of Brönsted and Lewis acid sites, especially for Lewis acid site, which caused the much higher B/L ratio than that of Z5-500. Interestingly, the composite structured Z5-inter showed much more Brönsted and Lewis acid sites than Z5-70 and Z5-500. But due to its larger amount of Lewis acid sites, the Z5-inter possessed the smallest $\mathrm{B} / \mathrm{L}$ ratio of 3.78. Although, Z5-inter-Al exhibited obvious decreased amount of acidity after the dealunimation of Z5-inter, the Brönsted acid sites were increased.

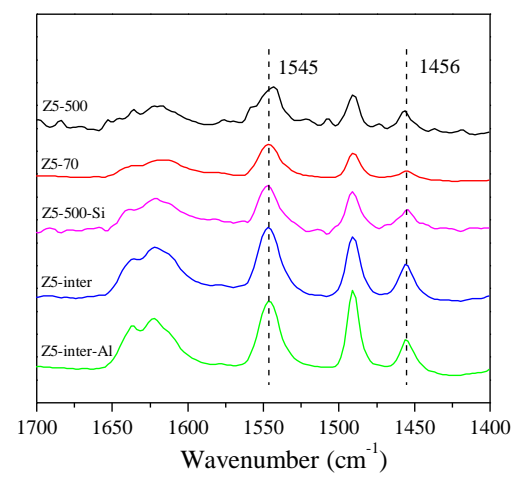

Fig. 7. Py-IR spectra of the samples at desorption temperature of $200{ }^{\circ} \mathrm{C}$.

Table 4. The distribution of Brönsted and Lewis acid sites on the different ZSM-5 zeolites.

\begin{tabular}{lccc}
\hline Samples & B (a.u./g) & L (a.u./g) & B/L \\
\hline Z5-500 & 39.35 & 12.82 & 4.05 \\
Z5-70 & 57.15 & 16.31 & 4.63 \\
Z5-500-Si & 32.94 & 4.75 & 9.15 \\
Z5-inter & 89.43 & 31.25 & 3.78 \\
Z5-inter-Al & 112.77 & 34.06 & 4.67 \\
\hline
\end{tabular}

${ }^{*} \mathrm{~B} / \mathrm{L}=1.32 \mathrm{IA}(\mathrm{B}) / \mathrm{IA}(\mathrm{L})$

\subsection{Catalytic performance}

The MTG activities of the five catalysts were studied at $405{ }^{\circ} \mathrm{C}, 1.0 \mathrm{MPa}$ and WHSV $=4.74$ $\mathrm{h}^{-1}$. During the reaction process, especially for the steady stage, methanol was basically converted into liquid hydrocarbons which were our mainly concerned products. Fig. 8 shows the yield of liquid hydrocarbons as a function of time on stream for the five catalysts. It was observed that for all the catalysts, the yield firstly increased, then underwent a steady period and finally decreased with time on stream. Among these catalysts, Z5-500 displayed the shortest lifetime of $54 \mathrm{~h}$. Its maximum yield was only $26.4 \%$ and then decreased quickly to $20.0 \%$ only after $40 \mathrm{~h}$. After desilication, Z5-500-Si presented an improved catalytic performance compared with parent Z5-500. The maximum yield was increased to $28.7 \%$ and the lifetime was prolonged to $86 \mathrm{~h}$. After reducing the crystal size, the maximum yield of Z5-70 reached up to $32.0 \%$ and catalytic lifetime 
was prolonged to $101 \mathrm{~h}$. While Z5-inter with intercrystalline mesopores showed a catalytic lifetime of $93 \mathrm{~h}$ and a maximum liquid hydrocarbons yield of $29.4 \%$.

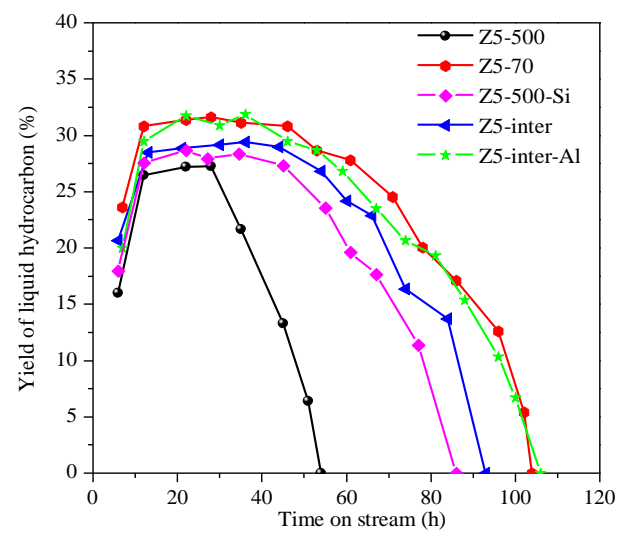

Fig. 8. Yield of liquid hydrocarbons on different ZSM-5 zeolites as a function of time on stream.

Table 5. The catalytic performance of different ZSM-5 zeolites.

\begin{tabular}{|c|c|c|c|c|}
\hline \multirow[b]{2}{*}{ Sample } & \multicolumn{2}{|c|}{ Liquid hydrocarbon } & \multirow{2}{*}{$\begin{array}{l}\text { Catalyst } \\
\text { lifetime (h) }\end{array}$} & \multirow{2}{*}{$\begin{array}{l}\text { Coke formation } \\
\operatorname{rate}^{\mathrm{b}}\left(10^{-3} \mathrm{~g} / \mathrm{g}_{\text {cat }} / \mathrm{h}\right)\end{array}$} \\
\hline & $\begin{array}{l}\text { Production }^{\mathrm{a}} \\
\left(\mathrm{g} / \mathrm{g}_{\mathrm{cat}}\right)\end{array}$ & $\begin{array}{l}\text { Yield of the stable stage } \\
\qquad(\%)\end{array}$ & & \\
\hline Z5-500 & 47 & 26.4 & 54 & 2.4 \\
\hline Z5-70 & 116 & 32.0 & 104 & 1.4 \\
\hline Z5-500-Si & 83 & 28.7 & 86 & 2.1 \\
\hline Z5-inter & 96 & 29.4 & 93 & 2.0 \\
\hline Z5-inter-Al & 112 & 31.7 & 106 & 1.5 \\
\hline
\end{tabular}

${ }^{\mathrm{a}}$ The liquid hydrocarbon production of the whole reaction process; ${ }^{\mathrm{b}}$ obtained by TG.

The catalytic stability is strongly related to the coke formation. As shown in Table 5, the coke formation rate of Z5-500 with the shortest longevity was the highest and reached $2.4 \times 10^{-3} \mathrm{~g} / \mathrm{g}_{\text {cat }} / \mathrm{h}$. But with the prolonging of lifetime, the coke formation rate of Z5-70 and Z5-500-Si were decreased to $1.4 \times 10^{-3}$ and $2.1 \times 10^{-3} \mathrm{~g} / \mathrm{g}_{\mathrm{cat}} / \mathrm{h}$, respectively. This indicated that decreasing the crystal size and introducing of mesopores decelerated the coke formation. Z5-inter has a composite structure of mesopores and nano-sized crystals, and presented a coke formation rate of $2.0 \times 10^{-3}$ $\mathrm{g} / \mathrm{g}_{\text {cat }} / \mathrm{h}$. This rate was higher than that of Z5-70, but lower than that of Z5-500-Si. Its catalytic lifetime also had the similar trend. As a whole, Z5-70 with the longest lifetime showed the lowest coke formation rate.

In fact, coke formation rate is strongly influenced by the external surface area, acidity and acid types. On one hand, large external surface area means more micropores openings exposed on the external surface and also shorter diffusion path length, which facilitates the migration of coke precursors within micropores to the external surface. On the other hand, strong acidity and high Brönsted acid sites density could accelerate the coke formation. Z5-500-Si possessed strong acidity, but its coke formation rate was lower than Z5-500, which was ascribed to its decreased 
Brönsted acid sites density and large external surface area. Although Z5-70 showed higher Brönsted acid sites density compared with Z5-500-Si, its weaker acidity and larger external surface area resulted in a slower coke formation rate than Z5-500-Si and Z5-500. By comparing these three catalysts, it could be interpreted that external surface area was significantly crucial for catalytic performance. The relationship between external surface area of these three catalysts and their catalytic lifetime was also investigated (Fig. 9). It can be seen that the catalytic lifetime increased with the increase of external surface area. This was further proved that reducing crystal size and introducing mesopores can decelerate the coke formation and thus prolong the catalytic lifetime.

It was noteworthy that Z5-inter presented an external surface area of $104 \mathrm{~m}^{2} / \mathrm{g}$, which was larger than Z5-70. However, its catalytic lifetime was shorter than Z5-70. According to recent research [28,29], aromatics (benzene, toluene, and xylene, BTX) were produced through polymerization and cyclization of light olefins, dehydrogenation of naphthenes, and the strong acidity of ZSM-5 catalyst usually promotes these reaction processes, resulting in a quick formation of coke and thus a fast catalyst deactivation. As shown in Table 3 and Fig. 6, Z5-inter presented a stronger acidity than others. This excessive acidity displayed an adverse effect on the catalytic lifetime. Besides, the high Brönsted acid sites density on Z5-inter also promoted the coke formation. After dealumination of Z5-inter, Z5-inter-Al only showed decreased acidity without obvious difference of morphology and textural property, which meant the similar diffusion property as Z5-inter (Fig. 6 and Table 3). For catalytic performance, its catalytic lifetime was prolonged from 93 to $106 \mathrm{~h}$ because of its low coke formation rate of $1.5 \times 10^{-3} \mathrm{~g} / \mathrm{g}_{\text {cat }} / \mathrm{h}$ (Table 5). However, despite its much larger external surface area than Z5-70, Z5-inter-Al exhibited similar lifetime as Z5-70. This limited improvement of lifetime on Z5-iner-Al was mainly due to its stronger acidity and its higher Brönsted acid sites density than Z5-70. Therefore, the acidity is also a crucial factor influencing the catalytic lifetime. An appropriate acidity and acid type were also essential for ZSM-5 to present good stability.

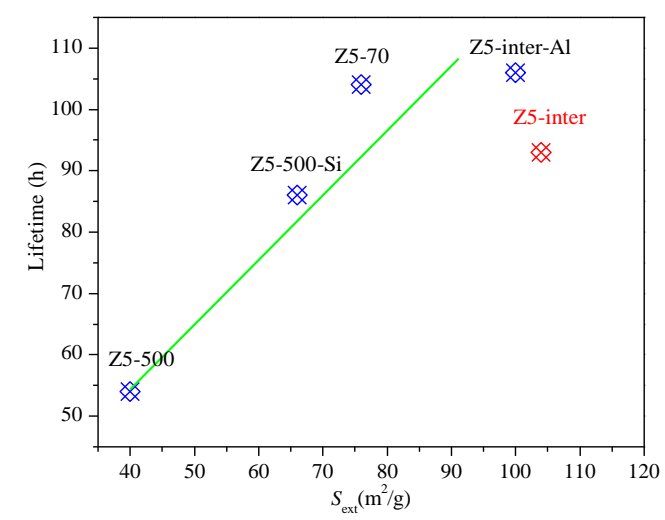

Fig. 9. Relationship between the lifetime and external surface area of ZSM-5 zeolites.

The total liquid hydrocarbons production $\left(\mathrm{g} / \mathrm{g}_{\mathrm{cat}}\right)$ of the prepared catalysts was also shown in Table 5. Obviously, the liquid hydrocarbons production was improved by decreasing the crystal 
size or introducing mesopores. Specifically, Z5-70 showed a higher liquid hydrocarbons production ability than $\mathrm{Z5}-500-\mathrm{Si}$, which can be ascribed to its large external surface and weak acidity. However, for Z5-inter with larger external surface area than Z5-70, its total hydrocarbons production was only $96 \mathrm{~g} / \mathrm{g}_{\mathrm{cat}}$, lower than Z5-70.After dealumination, total hydrocarbons production of Z5-inter-Alcatalyst was increased to $112 \mathrm{~g} / \mathrm{g}_{\text {cat }}$. Therefore, it could be concluded that ZSM-5 catalyst with large external surface area and low acidity strength were beneficial to the production of liquid hydrocarbons.

About product distribution, the liquid product in a mediocre time on stream of $24 \mathrm{~h}$ was collected and analyzed off-line, and the results were displayed in Fig. 10. Considering i-paraffin and aromatics were the main contributes for gasoline quality, the variation of the two components was mainly discussed. During the MTG reactions, i-paraffin was majorly produced by alkylation and the hydrogen transfer reactions of olefins, and aromatics were via cyclization and oligomerization of light olefins or dehydrogenation of naphthene [29]. As shown in Fig. 10, both reducing the crystal size and introducing mesopores facilitated the formation of i-paraffins. The fabrication of nano-sized ZSM-5 with intercrystalline mesoporous structure also increased the i-paraffins selectivity. Z5-500-Si has the biggest mesopore volume of $0.35 \mathrm{~cm}^{3} / \mathrm{g}$ that could provide large space for alkylation and isomerization of olefins and enhance mass transport of large molecules. Its selectivity of i-paraffins reached $25.6 \%$ and was the highest among these catalysts. Compared with Z5-500, the other four catalysts also showed a high selectivity of aromatics. Especially for Z5-inter, its aromatics selectivity reached $41.5 \%$ which was mainly due to its strong acidity (Fig. 6). This can be confirmed by the decreased aromatics selectivity for Z5-inter-Al with an obviously decreased acidity but a similar pore structure with Z5-inter.

Long catalytic lifetime, high yield of liquid hydrocarbons and proper selectivity of hydrocarbons are the main properties of ZSM-5 catalyst during its catalytic application. Based on the national standards of gasoline components [30], high aromatics content was needed for the increase of the gasoline quality. Decreasing crystal size was the direct and effective route to prolong the catalytic lifetime. However, the acidity of the nano ZSM-5 needs to be increased to promote the generation of aromatics. Also, the efficient synthesis method of nano ZSM-5 should be developed because its synthesis was far from industrial application due to some unavoidable challenges, such as the high-speed and time-consumption of centrifugation during its separation. On the contrary, introducing mesopores into micropore system of ZSM-5 could remarkably facilitate the molecule diffusion. Desilication is an effective way to fabricate mesopores and improves the yield of liquid hydrocarbons and catalytic lifetime. However, what deserves attention especially is how to controllably fabricate uniform mesopores. ZSM-5 with a composite structure formed with nano-sized crystals and rich mesopores will be a preferential choice. Intercrystalline mesoporous ZSM-5 prepared by salt-aided seed-induced route was easily obtained and had large external surface area, which can display good catalytic performance. Also, the selectivity of i-paraffin and aromatics on this catalyst were high, which was beneficial to 
gasoline quality. However, the strong acidity of this catalyst accelerated the formation of coke precursors and thereby led to quick catalyst deactivation. More work should be focused on the acidity modification for this composite structuralZSM-5to increase the stability, which can also further optimize the product selectivity.

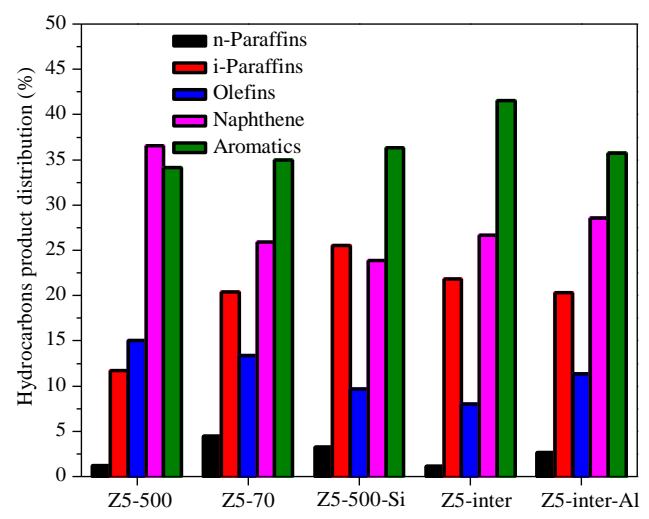

Fig. 10. Hydrocarbon component distribution on different ZSM-5 zeolites.

\section{Conclusions}

It was found that ZSM-5 with composite structure of intercrystalline mesopores and aggregated nano-sized crystals presented a large external surface area of $104 \mathrm{~m}^{2} / \mathrm{g}$ and a long catalytic lifetime of $93 \mathrm{~h}$. Considering the fact that its strong acidity could facilitate the coke formation, it was dealuminated to decrease the acidity and no obvious structure destruction was observed. After dealumination, the coke formation rate was decreased from $2.0 \times 10^{-3}$ to $1.5 \times 10^{-3}$ $\mathrm{g} / \mathrm{g}_{\mathrm{cat}} / \mathrm{h}$ and catalytic lifetime was prolonged to $106 \mathrm{~h}$. Large external surface area and weak acidity work together to prolong the catalytic lifetime of nano-sized ZSM-5 with intercrystalline mesopores. This special structural zeolite is a potentially excellent catalyst for methanol to gasoline reaction.

\section{Acknowledgments}

We gratefully appreciate the Science and Technology Foundation Platform Construction Project of Shanxi Province (No. 2015091009), the National Science Foundation for Young Scientists of China (No. 21606160), the Qualified Personnel Foundation of Taiyuan University of Technology (No. tyut-rc201454a) and School Fund of Taiyuan University of Technology (No.1205-04020202; No.1205-04020102).

\section{Reference}

[1] G. Busca, Chem. Rev. 107 (2007) 5366-5410.

[2] A. Corma, Chem. Rev. 97 (1997) 2373-2420.

[3] X. Wang, X. Gao, M. Dong, H. Zhao, W. Huang, J. Energy. Chem, 24 (2015) 490-496.

[4] U. Olsbye, S. Svelle, M. Bjørgen, P. Beato, T.V.W. Janssens, F. Joensen, S. Bordiga, K.P. Lillerud, Angew. Chem. Int. Edit. 51 (2012) 5810-5831.

[5] Y. Tao, H. Kanoh, L. Abrams, K. Kaneko, Chem. Rev. 106 (2006) 896-910.

[6] K. Egeblad, C.H. Christensen, M. Kustova, C.H. Christensen, Chem. Mater. 20 (2008) 946-960. 
[7] A. Petushkov, S. Yoon, S.C. Larsen, Microporous Mesoporous Mater. 137 (2011) 92-100.

[8] T.Q. Hoang, X. Zhu, L.L. Lobban, D.E. Resasco, R.G. Mallinson, Catal. Commun. 11 (2010) 977-981.

[9] T.V.W. Janssens, J. Catal. 264 (2009) 130-137.

[10]A.A. Rownaghi, J. Hedlund, Ind. Eng. Chem. Res. 50 (2011) 11872-11878.

[11] M. Firoozi, M. Baghalha, M. Asadi, Catal. Commun. 10 (2009) 1582-1585.

[12] S. Fathi, M. Sohrabi, C. Falamaki, Fuel. 116 (2014) 529-537.

[13] M. Bjørgen, F. Joensen, M. Spangsberg Holm, U. Olsbye, K.-P. Lillerud, S. Svelle, Appl. Catal. A. 345 (2008) 43-50.

[14] Y. Song, C. Sun, W. Shen, L. Lin, Catal. Lett. 109 (2006) 21-24.

[15] J.C. Groen, T. Bach, U. Ziese, A.M. Paulaime-van Donk, K.P. de Jong, J.A. Moulijn, J. Pérez-Ramírez, J. Am. Chem. Soc. 127 (2005) 10792-10793.

[16] H. Zhang, Z. Hu, L. Huang, H. Zhang, K. Song, L. Wang, Z. Shi, J. Ma, Y. Zhuang, W. Shen, Y. Zhang, H. Xu, Y. Tang, ACS. Catal. 5 (2015) 2548-2558.

[17] H. Zhang, Y. Ma, K. Song, Y. Zhang, Y. Tang, J. Catal. 302 (2013) 115-125.

[18] D.P. Serrano, J. Aguado, J.M. Escola, J.M. Rodriguez, A. Peral, J. Catal. 276 (2010) 152-160.

[19]H.Mochizuki, T. Yokoi, H. Imai, R. Watanabe, S. Namba, J.N. Kondo, T. Tatsumi, Microporous Mesoporous Mater. 145 (2011) 165-171.

[20] A.E. Persson, B.J. Schoeman, J. Sterte, J.E. Otterstedt, Zeolites. 14 (1994) 557-567.

[21] E.G. Derouane, S. Determmerie, Z. Gabelica, N. Blom, Appl. Catal. 1 (1981) 201-224.

[22] S.C. Larsen, J. Phys. Chem. C. 111 (2007) 18464-18474.

[23] W. Song, R.E. Justice, C.A. Jones, V.H. Grassian, S.C. Larsen, Langmuir. 20 (2004) 4696-4702.

[24] H. Zhu, Z. Liu, D. Kong, Y. Wang, X. Yuan, Z. Xie, J. Colloid. Interf. Sci. 331 (2009) 432-438.

[25] Q. Wang, S. Xu, J. Chen, Y. Wei, J. Li, D. Fan, Z. Yu, Y. Qi, Y. He, S. Xu, C. Yuan, Y. Zhou, J. Wang, M. Zhang, B. Su, Z. Liu, RSC. Adv. 4 (2014) $21479-21491$.

[26] H. Zhu, Z. Liu, D. Kong, Y. Wang, Z. Xie, J. Phys. Chem. C. 112 (2008) 17257-17264.

[27] K. Tarach, K. Góra-Marek, J. Tekla, K. Brylewska, J. Datka, K. MLekodaj, W. Makowski, M.C. Igualada López, J. Martínez Triguero, F. Rey, J. Catal. 312 (2014) 46-57.

[28] G.Q. Zhang, T. Bai, T.F. Chen, W.T. Fan, X. Zhang, Ind. Eng. Chem. Res. 53 (2014) 14932-14940.

[29] J. Li, Y. Wang, W. Jia, Z. Xi, H. Chen, Z. Zhu, Z. Hu, J. Energy. Chem, 23 (2014) 771-780.

[30] Y.Wang, R. Zheng, Y. Qin, J.Peng, M. Li, J. Lei, Y.Wu, M. Hu, S. Shuai, Fuel. 166 (2016) 543-552. 